\title{
VICTOR COUSIN, LA DOCTRINE ILLUSTRE QUE \\ LA FRANCE A DONNÉE AU MONDE ET D'OỦ EST SORTIE \\ LA PHILOSOPHIE MODERNE, LES PROGRĖS DES TEMIPS \\ ET LES BESOINS DU XIXÈ SIÈCLE
}

Patrice Vermeren

I

Y a-t-il une tradition philosophique française? Ou bien y-a-t-il seulement un usage français de la philosophie allemande ? Une tradition se donne une origine, réelle ou fantasmatique, dans laquelle elle inscrit les raisons de sa légitimité. Une tradition construit une orthodoxie par exclusion de ce qui n'est pas elle, en réduisant le divers à l'un. Une tradition véhicule un concept de la modernites, qui peut être soit pure répétition du même, soit invention du nouveau

Je suis parti de celte question, pour interroger la figure incontournable qui sert de référence à toute revendication nationale en matière de philosophie en France, mais qui est aussi mondialement revendiquée par tous les tenants de l'Universalité de la Raison, comme Victor Gómez Pin: Descartes. Pour en arriver à la naissance de la constitution de cette problénatique au début du xıxé siècle. Quelle place est-elle donnée à Descartes par Victor Cousin. dans son projet de ressourcer le spiritualisme en France contre la philosoplie du xvmè siècle (le "sensualismen), censée étre responsable des excès de la Révolution française, de la ruine de la morale et de la politique, et du sommeil philosophique dans lequel le pays semble être plongé au présent, dans le xıxè commençant, où la penséc paraît čtre, selon l'expression de Jouffroy, ajournée.

\section{Un Descartes sceptique}

Lorsque le jeune Victor Cousin remplace à la Sorbonne et à l'Ecole Normale Supérieure RoyerCollard, Condillac et les Idéologues avaient réduit le champ du débat philosophique en France à la seule question de l'origine des ides. L'institution philosophique se tient jusqu'en 1816 1817 dans "ce trou où l'on manquait d'air", encore selon l'expression de Jouffroy.

Laromiguière, dans ses leçons de philosophie professées à la Faculté des Lettres de Paris en 1811 et 1812, avait esquissé une critique timide du sensualisme en prétendant que les idées ne viennent pas toutes de la sensation, et que l'âme est active lorsque l'attention se porte sur l'objes. Mais la philosophic demeure chez lui réduite à l'analyse de l'entendement, qui commande ses questions et ses méthodes.

Sur sa tombe, Cousin dira de celui qui fut son premier prolesseur de philosoplie à l'Ecole Normale qu'il n'avait pas abandonné le système de Destutt de Tracy, mais qu'il avait le premier rélabilité l'intelligence dans l'activité, dans l'indépendance et dans la dignité qui lui appartiennent.

Royer-Collard, le second professeur de Cousin, avait été nommé par Napoléon dans la Cliaire d'histoire de la philosophie nouvellement créé pour lui dans le dessein de le débarrasser tout à fait des ldéologues en les tuant par le raisonnement. Il utilise les critiques ćcossais de Locke pour combattre la doctrine de Condillac. Mais son concept de la perception exterieure n'est pas ślaboré au-delà d"une réfutation élémentaire de la psychologia de la sensation transforméc. En lui dédicaçant son édition des Oemres de Descartıs en 1826, Victor Cousin donnera Royer-Collard comme le premier qui, dans une claire française, combattit la philosophic des sens et rélabilita Descartes. 
Mais c'est un jugement a posteriori. Car en 1815, la méfiance à l'égard du Cartésianisme est de mise. Lors de sa première leçon, le 7 décembre 1815, inaugurant un cours qui traite de la perception extérieure et de la réalité du monde en dehors de la conscience, soit la reprise de la question de Thomas Reid dans le droit fil de Royer-Collard, Cousin dit ceci:

\begin{abstract}
Il a été démontré avec la dernière rigueur que les théories élevées depuis 200 ans sur la question qui nous occupe sont essentiellement sceptiques; que la diversité qu'on rencontre dans les opinions des philosophes tombe seulement sur les formes du scepticisme: mais que toutes les renferment plus ou moins explicitement. Et qu'enfin la plilosophie moderne, fille de Descantes et mere de Hume, ne croit pas, ou n'a pas le droił de croire à l'existence du monde extérieur. D'où vient, Messieurs, pareille extravagance ? D'abord de la prétention de tout expliquer avec un seul principe, enfin de l'adoption de la conscience pour principe unique.
\end{abstract}

Il s'agit done de renouveler la condamnation du cartésianisme par Reid et Royer-Collard. La philosophie s'est enfermée dans le cercle du scepticisme à cause de Descartes qui a trop accordé au pouvoir d'examen de la conscience rationnelle.

Pour en sortir, il faut d'abord faire confiance au sens commun, véritable rempart contre le doute universel.

\title{
Leibniz, Je premier des philosophes allemands
}

Enfin, la troisième source de la philosophie nouvelle qui ne s'annonce que timidement en 1815 , avec Laromiguiëre et Royer-Collard, c'est Maine de Biran. Victor Cousin fréquente sa société philosophique à Auteuil avec Guizot, Royer-Collard, Ampère, Thureau, De Gérando. Et c'est à travers Maine de Biran qu'il va se mettre à lire Leibniz.

Déjà Madame de Staël, dans De l'Allemagne, avait montré que Leibniz inaugurait la nouvelle philosophie allemande et donnait à l'observation du fait de conscience la priorité sur le fait sensible: «Ce qui fonde da jamais sa gloire, c'est d'avoir su maintenir en Allemagne la philosophie de la liberté morale contre celle de la Fatalité sensuelle". "Tandis que le reste de l'Europe adoptait les principes qui font considérer l'âme comme passive, Leibniz fut avec constance le défenseur éclairé de la philosophie idéaliste».

Madame de Staël, on le sait, vit saisir son livre (1810) et le Préfet de Police lui signifie un ordre d'exil par ces termes: « II m'a paru que l'air de ce pays ne vous convenait point, et nous n'en sommes pas réduits à chercher des modèles chez les peuples que vous admirez. Votre dernier ouvrage n'est pas français ". Au demeurant, c'est chez elle que la jeunesse philosophique française apprend les noms de Kant, de Jacobi, de Fichte; de Schelling et de Herder.

Et done c'est chez Maine de Biran que Cousin ensuite apprend à lire Leibniz. Dans son cours de 1816, Cousin le qualifie de premier des philosophes allemands, en date comme en génie. "C'est lui qui le premier a nié la passivité de l'âme, démontré l'harmonic prétablic, l'expression de tout univers par chaque monade, l'existence de ses perceptions sourdes et obscures. Il est idéaliste, mais croit à l'existence des substances: on peut donc l'être sans être sceptique".

Dans l'article Leibniz de la Biographie Universelle de Michaud, Maine de Biran écrit (nous sommes en 1819), que l'auteur de la Monadalogie est le premier à avoir substitué l'idée de force à celle de substance. En aftirmant contre Descartes et ses disciples l'activité de loute substance créée, Leibniz montre que chaque monade contient en elle-mème le principe de tous ses changements, et exprime la multiplicité des existants et des possibles, soit Dieu et l'Univers. 
Je ne puis ici commenter le fait que Cousin dira que Maine de Biran a trop psyclologisé Leibniz, qu'il en a fait une lecture réductionniste qui exclut le non-moi, cause qui a ses lois aussi, que toute mon action ne peut pas clıanger. Et que s'il a bien replacé, face à la sensation, la volonte, il n'a pas fait de place à la raison impersonnelle, ce qui le conduit finalement au mysticisme.

\section{Descartes fondateur de J'école idéaliste moderne}

Treize ans plus tard, alors qu'il est passé par l'Allemagne et qu'il a nourri sa propre plitosophie de Kant, de Fichte, de Hegel et de Schelling, Victor Cousin revient dans son cours sur Leibniz. Et l'auteur de la Monadologie n'est plus seulement l'adversaire de Locke, il est aussi celui de Descartes : «Leibniz est le premier qui ait saisi le côté faible, le véritable vice du cartísianisme, à savoir la prédominance de l'idẻe de substance sur celle de cause,» C'est là l'un des meilleurs litres de gloire de Leibniz d'avoir perçu cette faille qui autorise les systèmes de Malebranche et de Spinoza, et l'on doit à Maine de Biran, un de nos compatriotes, de le lui avoir restitué.

Cousin dans ce texte donne explicitement à Leibniz la paternité de sa propre méthode, théorique et historique à la fois, et dont il décrit le caractère comme śtant de ne rien repousser et de tout comprendre pour employer tout.

Cette méthode, c'est l'écleclisme.

La plilosophie de Cousin se caractérise par deux philosophèmes qui sont:

1) La psychologie comme vestibule de la philosophie: il faut appliquer la méthode expérimentale de Bacon aux faits de conscience d'abord, plutôt qu'aux faits extérieurs. Conme on sait, Schelling y verra une contradiction posant comme un paradoxe de vouloir atteindre les vérités absolues par la méthode expérimentale.

2) L'idéc que les systèmes philosophiques possibles sont ipuisés: il y a quatre archétypes auxquels peuvent se réduire toutes les philosophies: l'idéalisme, le matérialisme. le scepticisme, le mysticisme. Ces figures paradigmatiques sont attesties depuis même la philosophie orientale. 11 est impossible de produire une philosophie nouvelle. II n'y a plus qu'à tracer le partage du vrai et du faux dans les systèmes passés. Donc la philosophie est aclıevée et on ne peut plus faire que de l'histoire de la philosophie. C'est exactement l'équivalent philosophique de la théorie politique de Guizot. Après les excès du monarchisme absolu et du démocratisme revolutionnaire, on ne saurait inventer une nouvelle forme de régime politique. Il faut donc un mixte, la monarchie constitutionnelle, avec un roi qui ne gouverne pas.

Done en 1828-1829, Cousin a naturalisé française la philosophie allemande. Il a puisé cliez ses maîtres Laromiguière: l'idée d'activité de l'âme; chez Royer-Collard: I'idée de perception; chez Maine de Birnn: l'idée de volonté. Et il crédite Leibniz de la paternité de son système. On sait qu'il a établi une édition de Descartes en onze volumes (1824-1826). Et dans la onzième leçon de son cours de 1829, il revient sur Descartes: c'est la fondation de l'école idéaliste moderne. Il cherche le point de dípart fixe et certain sur lequel peut s'appuyer la philosophie et le trotrve dans la pensée. Mais il commet une faute grave, un anachronisme évident dans l'histoire de la conscience, en ne plaçant pas sur la mėme ligne que l'existence de l'âme et celle de Dieu la conviction de l'existence du monde extérieur. Il ouvre ainsi la porte à Spinoza et à Malebranclie. «et là vous reconnaitrez les fruits lígitimes des principes du maitre").

\section{1}

C'est ici que je voudrais reperer la logique de la transformation qui va s'opérer dans la réfe. rence à Descartes. Et cette logique passe par Spinoza et la place de cette figure dans le cliamp 
agonistique de la nouvelle philosophie de Paris (expression de Stendlıal) devenue doctrine oflicielle de l'Université française face à l'Eglise Catholique.

\section{Le juif Spinoza: un mouni indien, un soufi persan}

Dans le cours qu'il prononce en 1829 à la Faculté des lettres de Paris sur l'histoire de la philosophie, Cousin écrit : "Au lieu d'accuser Spinoza d'athéisme, il faudrait bien plutôt lui adresser le reproche contraire ". Comment Spinoza peut-il partir de Descartes et de son être parfait et infini, et arriver finalement à l'unicité de la substance ? Cela ne peut être, selon Cousin, que par un vice de démonstration: "Quand l'homme n'a point été donné comme une cause volontaire et libre, mais comme un désir impuissant et comme une pensée imparfaite et finic, Dieu ou le modèle suprême de l'humanité ne peut être qu'une substance et non une cause, l'être parfait, infini, nécessaire, substance immuable de l'Univers et non sa cause productrice et créatrice". L'erreur de Spinoza est donc l'hypertrophie de la notion de substance, et l'abandon corrélatif de celle de cause. Cette erreur est symétrique de celle de Malebranclie, qui maintient la cause en Dieu et la degrade en l'homme, et finit par absorber I'homme en Dieu.

Spinoza est bien un disciple de Descartes, mais il va au-delà. Oủ donc? Vers le mysticisme, et non pas vers le matérialisme. C'est en tous cas ce que nous dit Cousin dans un texte rédigé en septembre 1836, tandis qu'il voyage en Hollande pour rédiger un rapport sur l'instruction publique de ce pays, mais aussi pour rechercher des fragments inedits de Descartes dans les papiers de Huyghens et visiter l'asile de tous les philosoples persécutés depuis deux siecles (Cousin luí-même s'était vu proposer par le roi des Pays-Bas d'entrer à son service en 1826). Et ne pouvant voir à Amsterdam la maison natale de Spinoza, le plilosophe français entre dans la synagogue des juifs portugais, s'y asseoit ; on y célèbre la fête de la réconciliation avec Dieu, mais lui ne pense qu'à Spinoza : "Quel que soit mon profond respect pour toute espèce de culte et en particulier pour le culte juif, j'avoue que dans cette synagogue je n'ai pu penser qu'à Spinoza. Assurément, je ne suis pas spinoziste; et après Leibniz et Maine de Biran, j’ai, dans mes leçons de 1829, parlé du système de Spinoza avec plus de sévérité que d'indulgence. En confondant le désir avec la volonté, Spinoza a détruit le véritable caractère de la personnalité humaine, et en général il a trop effacé la personnalité dans l'existence. Chez lui, Dieu, l'être en soi, l'éternel, l'infini, écrase trop le fini, le relatif, et cette humanité sans laquelle pourtant les attributs les plus profonds et les plus saints de la divinité sont inintelligibles et inaccessibles. Loin d'être un athée, comme on l'en accuse, Spinoza a terriblement le sentiment de Dieu, qu'il en perd le sentiment de l'homme». Plus loin, après avoir écrit que l'Ethique, malgré sa forme -Traité de géométrie, est au fond un hymne mystique, un élan et un soupir de l'äme vers celui qui, seul, peut dire légitimement: "Je suis celui qui suis", Cousin ajoute; "Spinoza, calommié, cxcommunié, présenté par les Juifs comme ayant abandonné leur foi, est essentiellement juif, et bien plus qu'il ne le croyait lui-mème. Oui, Spinoza est juif et quand il priait Jéhovah sur celte pierre que je foule, il le priait sincèrement dans l'esprit de la tradition judarque. Sa vie est le symbole de son système (...) Spinoza est un Mouni indien, un Soufi persan, un moine enthousiaste; et l'auteur auquel ressemble le plus ce pretendu athé est l'auteur inconnu del'/mitation de Jésus Christ». Je laisse de côté le thème de la vie vertueuse de Spinozn - argument systématiquement repris, de Bayle à Jean Reynaud, par tous ceux qui refusent de reconnaitre en Spinoza un monstre moral- Je voudrais tenter plutôt de saisir le sens de cette opération interprítative qui constitue Spinoza comme un mystique juif, en repérant dans l'actualité contemporaine ce à quoi clle s'oppose. 


\section{Le cher des panthéistes modernes}

Soit par exemple les Elénents de philosophie catholique de l'Abbé Combalot, publié en 1833, qui se réclament explicitement de de Bonald, de Maistre et Lamennais, et veut servir d'ouvrage de référence pour catholiciser la philosophie à l'usage de la jeunesse. Le prêtre, après avoir indjqué que c'est surtout dans Spinoza, Malebranclie et Berkeley que le cartésianisme devient méconnaissable, écrit: «Le juif Baruch Spinoza, né à Amsterdam en 1632 et mort en 1677. peut être regardé comme le chef des panthéistes modemes. Dans ce système, point de liberté divine, point de providence, point de liberté humaine, point de morale. En vain, Spinozn cherche à déduire la morale et l'obligation de la vertu, des notions les plus ćlevées de l'entendement; il n'y a ni devoir, ni vertu là où pẻse une étemelle, une invincible nécessité". Sept ans plus tard, et après l'abbś Bautain et son disciple l'abbé Goeschler, dans son Essai sur le panthéisme moderne, l'abbé Maret écrit: "Juif de naissance et élevé parmi les rabbins, Spinoza puisa done dans les doctrines de la cabale le germe de son système. Il revêtit d'un langage scientifique l'ancienne thêorie de l'émanation; et ce langage fut emprunté à l'ontologie de Descartes. Jamais le panthéisme n'avait été présenté sous une forme plus méthodique et plus rigoureusen. Toute la philosophie catholique du temps insiste sur la judaïté de Spinoza. Et elle lui assigne une place dans une tradition, quelle dénomme panthéiste, qui s'origine aux Védantistes, et dont la modernití est le fonds commun du rationalisme contemporain: Fichte, Schelling et Hegel en Allemagne, Cousin en France, Spinoza est la clef d'intelligibilité de ce système, parce qu'il lui donne sa forme achevée : présenté faussement comme l'héritier du cartesianisme (il donne à sa notion de substance d'où il tire son pantheisme, une définition qui ne concorde pas avec celle de Descartes), le spinozisme n'est en fait qu'un athéisme ou un matérialisme déguisé: il met l'homme à la place de Dieu. «L'athéisme consiste à nier Dieu, et à remplacer l'Etre des ètres par les forces aveugles de la nature. Le panthéisme appelle Dieu le grand tout de l'Univers; et ce grand tout, collection d'existences apparentes et illusoires, ne nous oflre en réalité qu'une abstraction, un substantif. De part et d'autr:, on refuse done à Dieu l'intelligence, la volonté, la liberté, la vie: on le nie".

Face à ces attaques - tout rationalisme est un panthêisme; or tout pantléisıne est un athéisme; donc l'éclectisme cousinien est un athéisme-, on perçoit mieux l'obstination de Victor Cousin à reconnaitre en Spinoza un mystique juif. Il peut alors distinguer entre deux panthésismes, l'un qui est identifiable avec le matérialisme et l'athéisme, l'autre, celui de l'auteur de l'Ethique, qui est un panthéisme spiritualiste qui dérive d'un sentiment essentiellement religieux à sa source. C'est cette tlièse que développe longuement Emile Saisset, son disciple, dans la Revue des Deux-Mondes en 1844: Spinoza, ce n'est pas l'absorption du fini dans l'infini, formule du théisme extravagant de l'école d'Elée, ni l'absorption de l'infini dans le fini, mais l'union nécessaire du fini et de l'inlini, la consubstantialité et la coéternité d'un univers toujours changeant et d'un dieu immuable. Il est fondamentalement l'homme qui incarne l'une des quatre figures archétypales de l'histoire de la philosophie: le mysticisme, à côté du matérialisme, du scepticisme et de l'idéalisme, qui ont épuisé le chamip des possibles et dans lequel il faut tailler la part du vrai et du faux, pour construire l'éclectisme, philosophic de l'âge de la monarchie constitutionnelle et de la réconciliation entre la mison et la religion.

Celte interprétation peut se légimiter de llerder, de Goethe, de Schlejermacher, de Novalis, pour les dépasser. Elle permet de maintenir, contre Spinoza. le principe de la liberté humatine, lié à celui de personnalité divine. Paul Janet en a montré la logique, qui est double: d'un point de vue métaphysique avec Maine de Biran et sa lecture psychologique de Leibniz, Cousin se donne comme point de départ l'acte personnel et individuel de la liberté humaine: il préserve le moi, la premiere et la plus assurée des existences; d'un point de vue politique, revendi- 
quant au nom du libéralisme l'héritage de la Révolution française et singulièrement la défense des droits de l'homme, il peut sauver l'individu d'une absorption par le tout, et résister au socialisme et au communisme, qui hantent les rêves des prolétaires et des réformateurs sociaux. Janet cite Tocqueville désignant le panthéisme comme un danger pour la démocratie:

İ mesure que l'individu se noie dans le semblable et l'identique, avec le progrès de l'égalité, on s'habitue à ne plus envisager les citoyens, pour ne considerer qute le peuple, on oublie les individus pour ne songer qu'à l'espice.

Le danger que représente le panthẻisme spinoziste importé d'Allemagne est celui d'un panthéisme naturaliste, qui absorbe l'homme dans la nature et fait de la nature l'être divin. Cousin, selon Janet, est censé y avoir opposé l'individu. De ce fait, il innocente Descurtes d'avoir engendré le matérialisme et l'athéisme: enjeu stratégique décisif, dès lors que l'on sait que dans les annèes 1840 , le chef de l'institution philosophique française abandonne toute référence à la philosophie allemande pour assigner au seul cartésianisme la paternité de la tradition philosophique française qu'il prétend avoir refondée.

De Descartes, père de la philosophie française, à Leibniz, dernier des philosophes cartésiens

Pour innocenter Descartes de la paternité d'un matérialisme athée, Cousin fait de Spinoza un mystique. Pour séparer sa philosophie de la référence allemande, il explique qu'il l'a toujours combattue. Et desormais, l'origine de la tradition qu'il a voulu fonder n'est plus Leibniz, mais devient Descartes.

En 1845, Cousin peut donc écrire dans la préface de ses Fragments de la philosophie cartésienne:

Quoi qu'en dise l'Angleterre, ce n'est pas Bacon, c'est Descartes qui est le pảre de la philosuphie française (...).

Au xviie siècle, l'Europ* entière suit la France, parce qu'en France il a paru un homme extraordinaire: Descartes en eflet a tout inventé. II est sans devancier, ou du moins sans modéle. L'école qu'il a fondée ne doit rien daucune inspiration étrangère. C'est un fruit du sol, c'est une oeurre qui, dans le fond et dans la forme, est profondement et exclusiveinent française, mâme encore plus, $s^{\prime} i l$ est pennis de le dire, que la poésie et les arts de cette grande et incomparable époque (...).

Pour ne parler ici que de Descantes, dis nos débuts et dans notre premier enseignement, de 1815 à 1820 , nous avons laautement défendu contre l'école écossaise et contre la philosophie allemande les principes de la psychologie et de la théodicée cantésienne. On peut voir dans la première série de nos cours une leçon de 1816 consacrée à démontrer, contre Reid, que le principe du cartésianisme, le tàmeux: «te pense donc je suis» ne renferme pas un cercle vicieux, et une autre leçon de 1820 duns laquelle, en réfutant le scepticisme de Kant, nous croyons avoir établi sans réplique que la preuve cartésienne đe l'existence de Dieu est pure de paralogisme et qu'elle est le fondement légitine de toute théodicke.

Plus tard, dans notre second enseignement, de 1828 a 1830 , nous ne nouss sommes pas bornés à glorifier partout le nom, le génie et la méthode de Deseartes; nous nous sornmes appliqués à mettre en lumière le caractère général, la suite, le progrès, les mérites, comme aussi les défauts, de l'ècole entière, depuis son fondateur jusqu'd Leibniz inclusivement. Car Leibniz est le dernier et le plus grand des cartésiens.

L'oeuvre de Descartes est cet « immuable monument, que le génie français a seul élevé et que seul il peut réparer et agrandir, sans toucher à ses fondements, selon les progrès du temps et les besoins du xixe siècle».

La tradition philosophique française a définitivement trouvé un père. Question pour une histoire politique de la philosophie? 


\section{BIBLIOGRAPHIE}

FRANCISQUE BOUILLIER: Histoire de la philosophie cartessienne, Paris, Delagrave, $3^{\circ}$ édition, 1868.

VICTOR COUSIN: Premiers essais de philosophie, Paris. Librairie Nouvelle, 1855, troisième édition revue et corrigée.

VICTOR COUSIN: Cours de I'histoire de la philosophie, Paris, Pichon et Didier, 18281829. Le cours de 1828: Introduction à /'histoire de la philosophie a été réédité dans le Corpus des ocuvres philosophiques de langue française, texte revu et corrige par Patrice Vermeren, Paris, Fayard, 1991.

VICTOR COUSIN: Fragntents de philosophie cartésienne, Paris, Charpentier, 1845.

RENÉ DESCARTES: Oewres, éditées par Victor Cousin, Paris, Piclion et Didier, $1824 / 1826,11$ volumes.

STÉPHANE DOUAILLER, ROGER-POL DROIT, PATRICE VERMEREN: Philosophie, France, $X I X^{\circ}$ siècle, Paris, Le Livre de Poche, 1994.

HUMBERTO GIANNINI, PIERRE-FRANÇOIS MOREAU, PATRICE VERMEREN (dir.): Spinoza et la politique, Paris, L'Harmattan, collection La philosophie en commun, 1996.

PAUL JANET: "Le spinozisme en France », Revue Philosophique, Paris, tome XIIl, révrier 1842, repris dans S.Douailler et alii, op.cit..

THÉODORE JOUFFROY: "Introduction aux oeurres philosophiques de M.RoyerCollard ", dans les Oewvres de Thomas Reid, Paris, A.Sautelet et Cie, Paris, 1828.

THÉODORE JOUFFROY: Mélanges philosophiques (1833) suivis de Nouveaux mélanges philosophigues (1842), texte revu et corrigé par Patrice Venneren, Corpus des Oeuvres philosophiques de langue française, Paris, Fayard, 1997.

PIERRE MACHEREY: "Les débuts philosophiques de Victor Cousin», dans Corpus, revue de philosophie, 99 avenue Ledru-Rollin, 75011 Paris, $n^{\circ} 18 / 191991$.

MAINE DE BIRAN: Oetnres, tome XIII, commentaires et marginalia par Joel Ganault, Paris, Vrin, 1990.

ABBE H.C.L. MARET: Essai sur le Pantheisme dans les sociétés modernes, Paris 1840. troisième édition Méquignon, 1845.

EMILE SAISSET: "De la philosophie du clergé", dans la Revue des Denur-Mfondes, 1844, repris dans Essais sur la philosophie ef la religion at. $\mathrm{x}^{\circ}$ siècle, Paris. Charpentier, 1845.

ANDRÉ SCHIMBERG: Les fragments philosophiques de Royer-Collard, Paris, Félix Alcan 1913.

PATRICE VERMEREN: "As aventuras da força activa em França», suivi de Maine de Biran: «Exposiçao da doutrina filosofica de Leibniz» con una prelácio de Victor Cousin, Analise $n^{\circ} 8$, Lisbonne, 1988.

PATRICE VERMEREN: "Victor Cousin, la institucion filosotica francesa y la doctrina maldita del judio Spinozan, Revista de Filosofia, Universidad de Chile, Santiago de Chile vol.XXXIX-XL 1992

PATRICE VERMEREN: Ifictor Cousin. Le jelt de la philosophie et de l'Etat. Paris, L'Harmattan, collection La philosophie en commun, 1995.

GERASSIMOS VOKOS: Philosophia kai ekpaideytike politike 10 kartesianismos sten galliké paideia ton $19^{\circ}$ aiöna), Athìnes, Lotos 1987. 